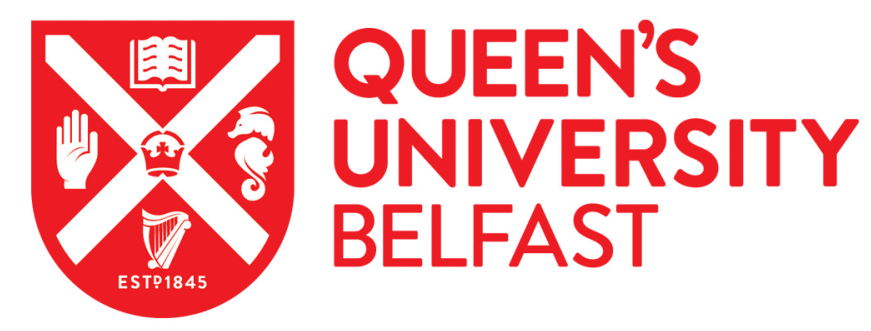

\title{
Influence of Bio-Isosteric Replacement on the Formation of Templating Methanol and Acetonitrile Solvates in Lophines
}

Kitchen, T., Melvin, C., Mohd Najib, M. N., Batsanov, A. S., \& Edkins, K. (2016). Influence of Bio-Isosteric Replacement on the Formation of Templating Methanol and Acetonitrile Solvates in Lophines. Crystal Growth and Design, 16(8), 4531-4538. https://doi.org/10.1021/acs.cgd.6b00655

Published in:

Crystal Growth and Design

Document Version:

Peer reviewed version

Queen's University Belfast - Research Portal:

Link to publication record in Queen's University Belfast Research Portal

Publisher rights

(C) 2016 American Chemical Society.

This work is made available online in accordance with the publisher's policies. Please refer to any applicable terms of use of the publisher.

\section{General rights}

Copyright for the publications made accessible via the Queen's University Belfast Research Portal is retained by the author(s) and / or other copyright owners and it is a condition of accessing these publications that users recognise and abide by the legal requirements associated with these rights.

Take down policy

The Research Portal is Queen's institutional repository that provides access to Queen's research output. Every effort has been made to ensure that content in the Research Portal does not infringe any person's rights, or applicable UK laws. If you discover content in the Research Portal that you believe breaches copyright or violates any law, please contact openaccess@qub.ac.uk. 


\title{
The influence of bio-isosteric replacement on the
}

\section{formation of templating methanol and acetonitrile}

\section{solvates in lophines}

Thomas Kitchen, ${ }^{a}$ Connor Melvin, ${ }^{a}$ Mohd Nadzri Mohd Najib, ${ }^{a}$ Andrei S. Batsanov ${ }^{b}$ and Katharina Edkins ${ }^{a *}$

a School of Medicine, Pharmacy and Health, Durham University Queen's Campus, University Boulevard, Stockton-on-Tees, TS17 6BH, United Kingdom; email:

katharina.fucke@durham.ac.uk

${ }^{\mathrm{b}}$ Department of Chemistry, Durham University, South Road, Durham, DH3 1LE, United Kingdom.

Keywords: bio-isosteres, solvate, crystal structure, triphenylimidazole

\begin{abstract}
Bio-isosteric replacement is a frequently used tool in medicinal chemistry. Whilst the pharmacological activity is not influenced by the exchange of substituents, the solid-state characteristics and formation of different crystal forms may well be altered dramatically,
\end{abstract}


jeopardising the processability and safety of the drug compound. In this study we investigate a series of triphenylimidazole (TPI) derivatives as model compounds with the bio-isosteric exchange of only one halogen position $(\mathrm{F}, \mathrm{Cl}, \mathrm{Br}, \mathrm{I})$. Crystallisation from two industrially used solvents (methanol and acetonitrile) reveals solvate formation of all TPIs, for which the basic hydrogen bonded motif does not change. The three-dimensional packing depends on the size of the substituent and changes from fluoro- to chloro- and bromo-substitution but remains the same for the larger iodo-substituent. From acetonitrile, only F-TPI and Cl-TPI form an isomorphic channel solvate, which in both cases desolvates reversibly to an isomorphic crystal form. Due to the halogen atom lining of the channels, bromine and iodine are too large to generate a stable packing. This study illustrates the importance of understanding the influence of bio-isosteric substitution on the solid-state, in order to best utilize this common tool.

\section{Introduction}

The development of new pharmaceutical compounds to treat emerging and pandemic diseases such as ebola or long-standing problems such as bacterial infections is amongst the key topics of pharmaceutical research. It demands a steady stream of novel pharmaceutical compounds to tackle the disease in question. One of the tools used in the area of medicinal chemistry is bioisosteric replacement, which is the replacement of substituents of a lead molecule with other chemical groups without changing the overall biological activity. ${ }^{1,2}$ This enables the medicinal chemist to improve specific properties, such as solubility, crystallinity, metabolic stability etc., which subsequently will improve the overall processability of the new drug compound. This concept has been applied to as diverse drug classes as cannabinoids ${ }^{3}$ and HIV antivirals. ${ }^{4}$ The selection of replacement groups is nowadays heavily helped by in silico studies and property predictions. $^{5-7}$ 
Even though this approach has been used for over 60 years, ${ }^{8}$ the implications of bio-isosteric replacements of substituents, which sometimes differ vastly in size and/or electronics, on the crystallisation properties of the new molecule have been widely ignored. Small organic molecules show a high probability of crystallising in multiple crystal forms, which may differ in physico-chemical characteristics such as melting point or solubility. ${ }^{9-12}$ The latter characteristic is especially important for pharmaceuticals, as solubility and especially water solubility is directly linked to bioavailability of the drug compound and thus its pharmacological activity in patients. In addition to crystal forms that contain the desired molecule, solvates incorporate solvent molecules from the crystallisation process. ${ }^{13}$ Solvates can be interpreted as intermediates formed during the crystallisation process, ${ }^{14,15}$ and can introduce traces of unwanted solvent into the pharmaceutical product, which can prove harmful or toxic to the patient. Thus, the levels of residual solvent in these applications are regulated. ${ }^{16}$ On the other hand, solvates represent a route to obtain novel unsolvated crystal forms, which are not accessible by direct crystallisation. ${ }^{17-22}$

The inclusion of solvent molecules into crystal forms has been widely studied, not only in the pharmaceutical field. The influence of solvate formation has been studied in a co-crystal system that has been crystal-engineered to undergo photodimerisation. It was found that the inclusion of acetonitrile leads to a molecular arrangement allowing photodimerisation, whilst the methanol solvate hinders this. ${ }^{23}$ In other studies, inclusion has been utilised to separate xylenes ${ }^{24}$ showing a clear preference of one guest over another, ${ }^{25}$ or to store volatile guests. ${ }^{26,27}$ Inclusion complexes have recently become focus of attention to study the incorporated molecules, either in an unusual conformation, ${ }^{28}$ or to obtain structural information of a hard to crystallise compound. ${ }^{29,30}$ 
Considering the variation in size and interaction potential in bio-isosteric groups, the choice of replacement group will influence the crystal structure of the substances, as has recently been shown for a series of trityl compounds. ${ }^{31}$ In addition, change of halogen substituents from chloro via bromo to iodo on phloroglucinol has been shown to influence the incorporation mode of water into the crystal structure. ${ }^{32} \mathrm{We}$ are thus systematically investigating the influence that the change of only one substituent has on the crystallisation behaviour and polymorphism of model compounds. ${ }^{33}$ In order to reduce the possibility of strong interactions between the molecules, we use 2,4,5-triphenylimidazoles (lophine, TPI, Figure 1) as our model, which can only donate and accept one hydrogen bond and show thus a reduced complexity compared to pharmaceutically used compounds. Lophine derivatives are used as core structures in medicinal chemistry, ${ }^{34}$ as well as for photophysical applications. ${ }^{35-37}$

During our work on the polymorphism of halogenated TPIs, we investigated a series of 2-(4'p-X-phenyl)-4,5-diphenylimazoles (X-TPI; $\mathrm{X}=\mathrm{F}, \mathrm{Cl}, \mathrm{Br}$, and I). These substituents are frequently exchanged for bio-isosteric replacement, ${ }^{38}$ and do not significantly change the overall electronics of the resulting compounds. However, the substituents vary considerably in size, with F having the smallest van-der-Waals radius with $1.47 \AA, \mathrm{Cl}$ with $1.75 \AA$, Br with $1.85 \AA$ to I with $1.98 \AA$. In the present study we report our results of crystallisation experiments with two standard solvents, methanol and acetonitrile, which are frequently used in pharmaceutical processes. 


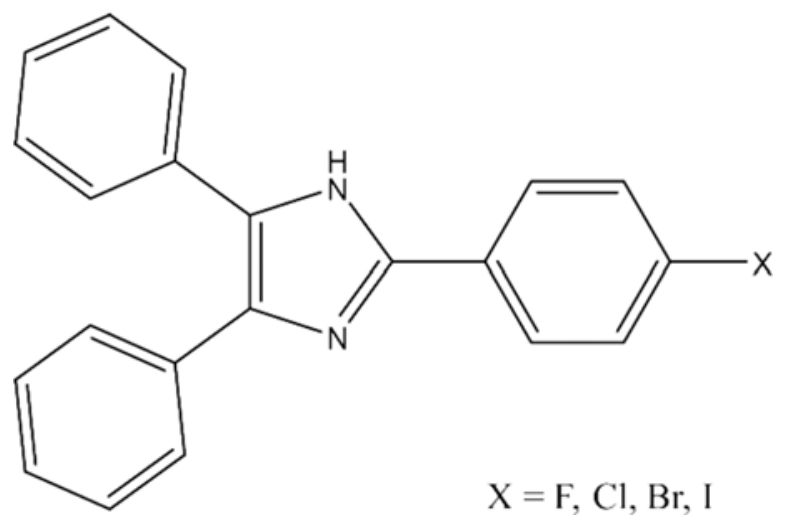

Figure 1. Molecular structure of 2,4,5-triphenylimidazole (TPI) derivatives (lophines) used in this study.

\section{Experimental section}

\section{Materials}

Benzil, $p$-halogenbenzaldehyde $(\mathrm{F}, \mathrm{Cl}, \mathrm{Br}, \mathrm{I})$, ammonium acetate, potassium carbonate and all organic solvents were purchased from Sigma Aldrich and used without further purification.

\section{Methods}

\section{$\underline{\text { Synthesis }}$}

All TPIs were synthesised using the literature method. ${ }^{39}$ Equimolar amounts of benzil and substituted benzaldehyde were suspended in acetic acid with 10-fold amount of ammonium acetate. The suspension was then heated in a microwave to $180{ }^{\circ} \mathrm{C}$ for $5 \mathrm{~min}$ and cooled to room temperature. The resulting solution was then poured into ice water and neutralised with $\mathrm{K}_{2} \mathrm{CO}_{3}$ forming a beige precipitate. This was collected by filtration and washed with $\mathrm{H}_{2} \mathrm{O}$ before recrystallisation.

\section{Crystallisation methods}

Approximately $10-20 \mathrm{mg}$ compound was used in each crystallisation experiment. For slow cooling and fast cooling experiments, sufficient solvent was added to produce a hot-saturated 
solution. These were then left to cool to room temperature in an insulating wooden block (slow cooling) or quenched in an ice and water bath (fast cooling). For evaporation, the material was dissolved in sufficient solvent to produce an undersaturated solution at room temperature and let stand to evaporate. Precipitation was conducted by producing a hot saturated solution and adding at least double the volume of cold (room temperature) diethyl ether as anti-solvent. The crystals forming were immediately removed from solution in case of the fast cooling and precipitation experiments, whilst evaporation and slow cooling experiments were left for one day before analysis.

\section{Differential scanning calorimetry}

Differential scanning calorimetry (DSC) was performed with a Q2000 (TA instruments, New Castle, DE, USA). Approximately 1 to $3 \mathrm{mg}$ of sample were accurately weighed (microbalance, Sartorius, Göttingen, Germany) into sealed Al pans and submitted to a ramp scan in the range from 25 to $370{ }^{\circ} \mathrm{C}$ with a heating rate of $10{ }^{\circ} \mathrm{C} \min ^{-1}$. Dry nitrogen was used as the purge gas (purge: $50 \mathrm{~mL} \mathrm{~min}^{-1}$ ). The instrument was calibrated for temperature and energy in the respective temperature range with pure indium (purity $99.999 \%$, m.p. $156.6^{\circ} \mathrm{C}$, heat of fusion $28.45 \mathrm{~J} \mathrm{~g}^{-1}$ ). Thermogravimetric analysis

Measurements were performed with a Q500 thermogravimetric system (TA instruments, New Castle, DE, USA). Samples of approximately 2 to $6 \mathrm{mg}$ were accurately weighed into $50 \mu 1$ platinum pans and subjected to non-isothermal TGA at a heating rate of $10{ }^{\circ} \mathrm{C} \mathrm{min}^{-1}$. Nitrogen was used as purge gas at a purging rate of $60 \mathrm{ml} \mathrm{min}^{-1}$.

\section{$\underline{\text { X-ray diffractometry }}$}

Powder X-ray diffraction patterns were recorded using an Empyrean diffractometer (Panalytical, Almelo, Netherlands) in Bragg-Brentano geometry with $\mathrm{Cu}-\mathrm{K} \alpha$ radiation, graphite 
monochromator, $0.2 \mathrm{~mm}$ fixed Soller slits and PIXcel detector. The X-ray tube was operated at $40 \mathrm{kV}$ and $40 \mathrm{~mA}$, while the samples were prepared on a low-background silicone slide sample holder as dry powder.

Single crystals of F-TPI and Br-TPI methanol solvates were picked, coated in perfluoro polyether oil and mounted on MiTeGen sample holders. These were placed directly into a precooled cryostream on a Bruker (Karlsruhe, Germany) D8 Quest diffractometer using Mo-Ka radiation $(0.71073 \AA)$. Single crystals of Cl-TPI acetonitrile solvate were picked, coated in perfluoro polyether oil and mounted on MiTeGen sample holders. The diffraction experiment was carried out on a Rigaku (Sevenoaks, UK) Saturn 724+ diffractometer at Station I19 of the Diamond Light Source synchrotron (undulator, $\lambda=0.6889 \AA$, $\omega$-scan, $1.0^{\circ}$ per frame). The data from a 4-component non-merohedral twin were processed using Bruker Apex-II software, deconvoluted using CELL_NOW ${ }^{40}$ and corrected for absorption using TWINABS 2012/1. ${ }^{41}$ The structure was solved using Olex.solve ${ }^{42}$ and refined using ShelXL. ${ }^{43}$ All non-hydrogen atoms could be located from Fourier difference maps and were refined anisotropically. Hydrogen atoms were calculated as geometrical riding models on the heavy atom and refined isotropically.

\section{Results and discussion}

\section{Methanol solvates}

Crystallisation of all TPI derivatives from methanol yielded a crystal form containing solvent from crystallisation. The analysis of the respective TG thermograms (Figure 2a) reveals a weight loss corresponding to 1 mol methanol per mol TPI (Table 1) for all crystal forms. The weight loss of F-TPI starts almost immediately with a slow zero-order loss of solvent, which is most likely the evaporation of trapped and surface-bound solvent. The weight loss speeds up at temperatures above $60{ }^{\circ} \mathrm{C}$ resulting in a one-step solvent release. The solvates of $\mathrm{Cl}$-, $\mathrm{Br}$ - and I- 
TPI show slightly higher stability with the start of the weight loss not below $70{ }^{\circ} \mathrm{C}$. Cl-TPI shows a single mass loss step comparable to F-TPI, whilst Br-TPI and I-TPI show multiple steps. All desolvation events are in the same temperature range as the boiling point of methanol, which indicates the high stability of these crystal forms.

The corresponding DSC thermograms (Figure 2b) show the desolvation as endothermic events in temperatures regions comparable to those detected by TG. Interestingly, Br-TPI and I-TPI show double peaks, which might be due to partial desolvation followed by melting of the remaining intact solvated crystal form, i.e. passing of the peritectic. This observation fits well with the two-step desolvation detected by TG. All TPI solvates desolvate into a stable crystalline form, which melts at temperatures above $250^{\circ} \mathrm{C}$.
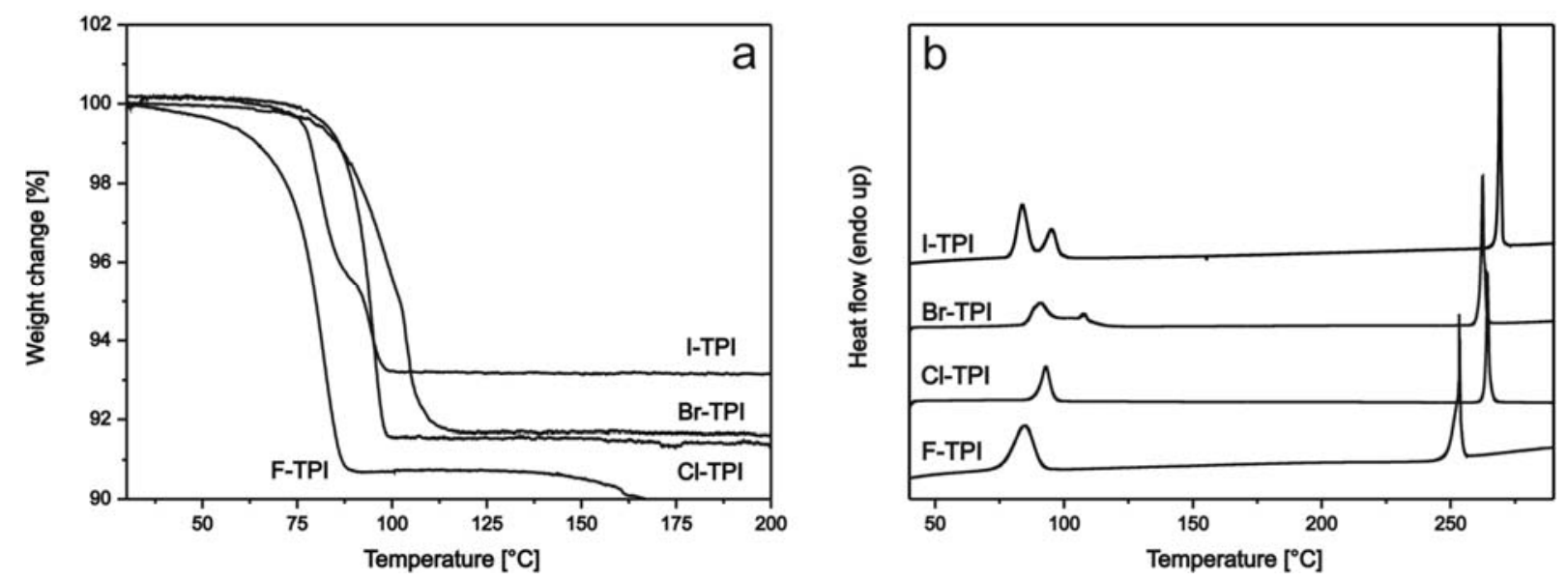

Figure 2. Thermoanalytical data of the four TPI methanol solvates: a) TG thermograms and b) DSC thermograms. 
Table 1. Weight loss of the methanol solvates as determined by TG analysis

\begin{tabular}{lccccc}
\hline & $\begin{array}{c}\text { Weight loss } \\
\text { range }\left[{ }^{\circ} \mathrm{C}\right]\end{array}$ & $\begin{array}{l}\text { weight loss } \\
{[\%]}\end{array}$ & $\begin{array}{c}\text { molar } \\
\text { ratio }\end{array}$ & $\begin{array}{c}\text { calc. weight loss } \\
\text { monomolar }[\%]\end{array}$ & $\begin{array}{c}\text { melting unsolvated } \\
\text { form (onset) }\left[{ }^{\circ} \mathrm{C}\right]\end{array}$ \\
\hline F-TPI & $52-92$ & 9.31 & 1.007 & 9.250 & 252.9 \\
Cl-TPI & $70-102$ & 8.42 & 0.949 & 8.831 & 262.7 \\
Br-TPI & $70-118$ & 8.32 & 1.063 & 7.867 & 261.2 \\
I-TPI & $73-100$ & 6.80 & 0.962 & 7.053 & 268.0 \\
\hline
\end{tabular}

The methanol solvates of F-TPI and Br-TPI could be crystallised in sufficient quality to obtain single crystal structures. The crystallographic data of these two crystal forms are listed in Table 2. Whilst the most common packing motif of imidazole derivatives is the hydrogen bonded chain, ${ }^{35,}{ }^{44}$ this motif is broken in the case of the methanol solvates. In both crystal forms, the methanol molecule intercalates into the chains accepting one hydrogen bond from one imidazole and donates a second to the next imidazole (Figure 3a). The TPI molecules are oriented counterparallel whilst the chains show a zig-zag ribbon like conformation with the edge being formed by the methanol molecules (Figure 3b). These then stack on top of each other. The next stacks are connected through weaker $\mathrm{C}-\mathrm{H} \cdots \pi$ interactions of adjacent non-substituted phenyl rings. Even though the bromine substituents are closer than twice their van der Waals radius (distance: 3.5472(5) $\AA$ with $2 \times$ vdW radius: $3.7 \AA$ ), the orientation of the TPI molecules and thus the geometry of the $\mathrm{Br}-\mathrm{Br}$ interaction does not directly point towards halogen bonding and thus this interaction is less likely to be a driving force of this packing motif. 

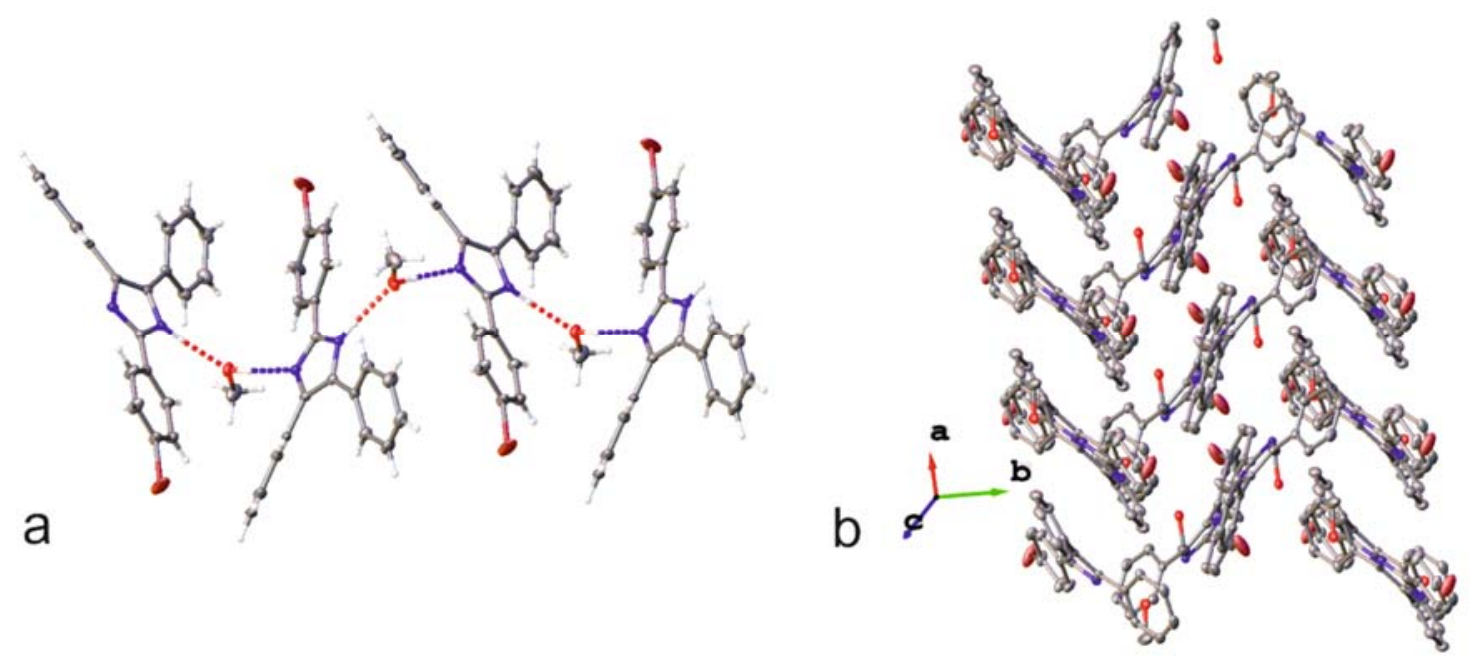

Figure 3. Molecular structure of Br-TPI methanol monosolvate: a) hydrogen bonded chains with intercalated methanol and b) stacking of the chains in the crystal structure, hydrogen atoms are omitted for clarity. Colour code: carbon (grey), hydrogen (light grey), nitrogen (blue), oxygen (red), bromine (brown).

Interestingly, the packing of the stacks is different for F-TPI compared to Br-TPI. Whilst the fluoro-substituent is small enough to allow the phenyl rings to properly interlock giving a dense packing, the bromine atom is too large for this motif. This results in two distinct changes of the packing: firstly, adjacent layers in the crystallographic $a$ direction of Br-TPI move further apart and secondly, the layers shift in the crystallographic $b$-direction of Br-TPI so that the bromine atoms orient along channels. This results in a higher symmetry of the crystal structure as well as a slightly different long range packing. Whilst F-TPI methanol solvate crystallises in identical layers, i.e. AAAA, Br-TPI methanol solvate shows alternating layers, i.e. ABAB, as can be seen by the shift in opposite directions of the $\mathrm{Br}-\mathrm{Br}$ pairs (Figure 4). This correlation is reminiscent of polytypism, even though these two crystal forms change in their chemical entities ( $F$ vs. Br substitution) and thus the term ca, strictly speaking not be used. ${ }^{45}$ 
The crystallographic symmetry of the F-TPI methanol solvate generates disorder in the hydrogen bonded chains. The asymmetric unit contains three methanol molecules, of which two lie on special positions on the 2-fold axis. Thus, their OH-groups show disorder over two positions generating disorder in the position of the proton of the imidazole ring. Even though the third methanol molecule can be refined without disorder due to its general position, the hydrogen bonded chain forces its $\mathrm{OH}$-group to be disordered as well. Since refinement of the structure in $P 1$ resolves the disorder, it can be assumed that the crystallographic symmetry would be broken if hydrogen atom positions could be located accurately, e.g. by using neutron diffraction.
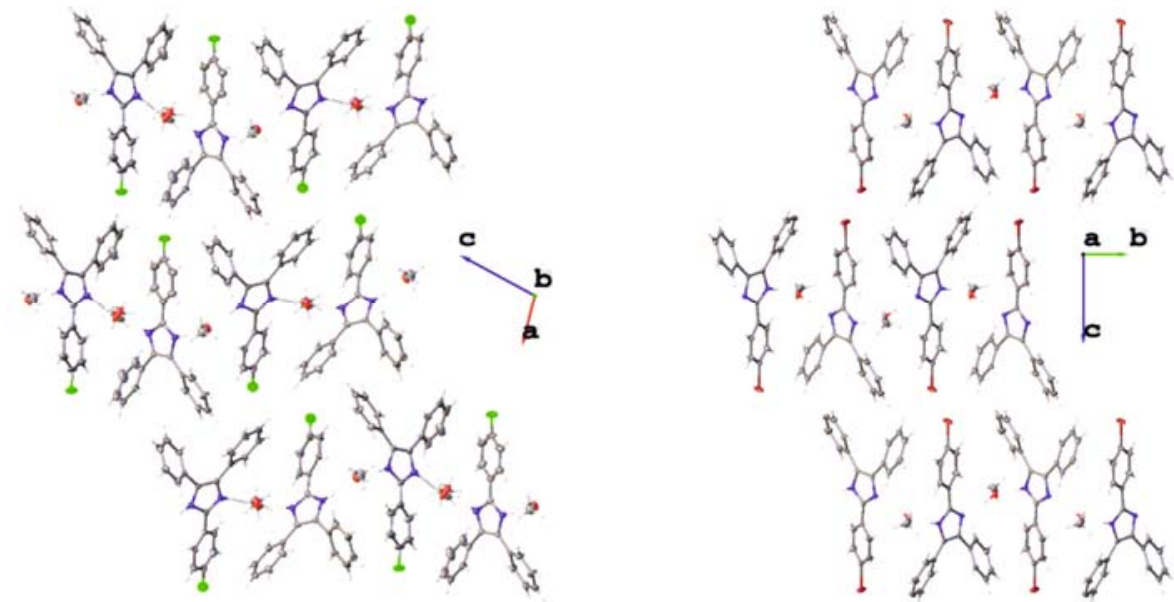

Figure 4. Molecular packing in the methanol solvates of F-TPI vs. Br-TPI.

Table 2. Crystallographic data of the methanol solvates of F-TPI and Br-TPI as well as the acetonitrile solvate of Cl-TPI as determined by X-ray single crystal diffraction.

\begin{tabular}{llll}
\hline Crystal form & F-TPI x MeOH & Br-TPI x MeOH & Cl-TPI x AcN \\
\hline Formula & $\mathrm{C}_{21} \mathrm{H}_{15} \mathrm{~N}_{2} \mathrm{~F} \mathrm{x} \mathrm{CH} 4 \mathrm{O}$ & $\mathrm{C}_{21} \mathrm{H}_{15} \mathrm{~N}_{2} \mathrm{Br} \times \mathrm{CH}_{4} \mathrm{O}$ & $\mathrm{C}_{21} \mathrm{H}_{15} \mathrm{~N}_{2} \mathrm{Cl}$ \\
& & & x $0.25 \mathrm{C}_{2} \mathrm{H}_{3} \mathrm{~N}$ \\
$M_{\mathrm{r}}\left(\mathrm{g} \mathrm{mol}^{-1}\right)$ & 346.39 & 407.31 & 341.06
\end{tabular}




\begin{tabular}{|c|c|c|c|}
\hline Crystal system & monoclinic & monoclinic & monoclinic \\
\hline Spacegroup & $P 2$ & $P 2_{1} / c$ & $P n$ \\
\hline$T(\mathrm{~K})$ & 120 & 120 & 100 \\
\hline$a(\AA)$ & $13.164(1)$ & $5.9477(3)$ & $8.854(1)$ \\
\hline$b(\AA)$ & $5.9105(6)$ & $11.6799(7)$ & $19.875(3)$ \\
\hline$c(\AA)$ & $23.800(2)$ & $27.003(2)$ & $19.924(3)$ \\
\hline$\alpha$ & 90 & 90 & 90 \\
\hline$\beta$ & $104.421(3)$ & $91.541(2)$ & $93.712(2)$ \\
\hline$\gamma$ & 90 & 90 & 90 \\
\hline$V\left(\AA^{3}\right)$ & 1793.4(3) & $1875.2(2)$ & $3498.6(9)$ \\
\hline$Z$ & 4 & 4 & 8 \\
\hline \multicolumn{2}{|c|}{ Density (calc, $\mathrm{g} \mathrm{cm}^{-3}$ ) } & 1.283 & $1.443 \quad 1.295$ \\
\hline Abs coeff. $\left(\mathrm{mm}^{-1}\right)$ & 0.087 & 2.205 & 0.208 \\
\hline $\mathrm{F}(000)$ & 728 & 831 & 1420 \\
\hline \multicolumn{2}{|c|}{ Crystal size $\left(\mathrm{mm}^{3}\right) 0.237 \times 0.231$} & $0.619 \times 0.188$ & $0.5 \times 0.02$ \\
\hline & x 0.12 & x 0.093 & x 0.02 \\
\hline $\begin{array}{l}\Theta \text { range for } \\
\text { data collection }\end{array}$ & $2.066-25.999$ & $1.90-26.00$ & $0.993-24.415$ \\
\hline \multirow[t]{3}{*}{ Index ranges } & $-15 \leq \mathrm{h} \leq 15$ & $-7 \leq \mathrm{h} \leq 7$ & $-10 \leq \mathrm{h} \leq 10$ \\
\hline & $-7 \leq \mathrm{k} \leq 7$ & $-14 \leq \mathrm{k} \leq 14$ & $-23 \leq \mathrm{k} \leq 23$ \\
\hline & $-29 \leq 1 \leq 29$ & $-34 \leq 1 \leq 34$ & $-23 \leq 1 \leq 23$ \\
\hline Reflections col. & 20125 & 51111 & 34297 \\
\hline Independent refl. & 7045 & 3697 & 34297 \\
\hline \multicolumn{2}{|c|}{ Refinement method } & \multicolumn{2}{|c|}{ full-matrix least-squares on $F^{2}$} \\
\hline $\begin{array}{l}\text { Data/restraints/ } \\
\text { parameter }\end{array}$ & $7045 / 1 / 477$ & $3697 / 0 / 236$ & $34287 / 2 / 896$ \\
\hline \multicolumn{2}{|c|}{ Goodness of fit on $F^{2}$} & 1.021 & 1.03121 .012 \\
\hline
\end{tabular}




\begin{tabular}{rlll}
$\mathrm{R}$ indices $[\mathrm{I}>2 \sigma \mathrm{I}]$ & $\mathrm{R}_{1}=0.0583$ & $\mathrm{R}_{1}=0.0330$ & $\mathrm{R}_{1}=0.0444$ \\
$\mathrm{wR}_{2}=0.1091$ & $\mathrm{wR}_{2}=0.1127$ & $\mathrm{wR}_{2}=0.1043$ \\
$\mathrm{R}$ indices (all data) $\mathrm{R}_{1}=0.1137$ & $\mathrm{R}_{1}=0.0427$ & $\mathrm{R}_{1}=0.0509$ \\
$\mathrm{wR}_{2}=0.1260$ & $\mathrm{wR}_{2}=0.1255$ & $\mathrm{wR}_{2}=0.1068$ \\
\hline
\end{tabular}

Since only two of the four solvates could be crystallised for single crystal diffraction, it is difficult to make definite statements about the packing of the other two crystal forms. It can be assumed, however, that the hydrogen-bonded chains persist in all four crystal forms. This is corroborated by the very similar desolvation temperatures of all four crystal forms indicating that the desolvation process has a comparable activation energy, i.e. the energy necessary to break the interactions between the solvent and the host molecules. If the basic packing motif would change drastically between the four solvates, a wider variation of desolvation temperatures would be anticipated alongside different desolvation kinetics.

The powder X-ray diffractograms of all four TPI derivatives show certain similarities (Figure 5). Whilst Br-TPI and I-TPI can be regarded as isostructural, which can be detected as a shift of the peak positions without changing the overall pattern, there is no similarity between these two, F-TPI and Cl-TPI. It can thus be assumed that the packing of Br-TPI is the most efficient for the compounds with large substituents in this series, whilst the smaller ones can pack in different arrangements of the basic hydrogen bonded planes. 


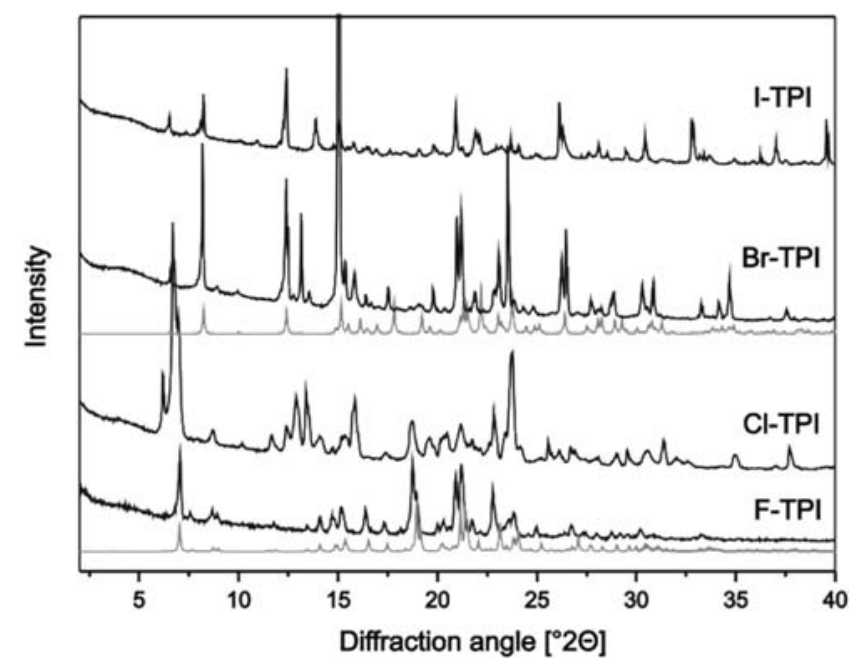

Figure 5. Powder X-ray diffractograms of the methanol solvates of the four TPI derivatives. Calculated patterns of the single crystal structures in light grey.

Upon desolvation, all methanol solvates transform into an unsolvated crystal phase (Figure 6). All of these show low crystallinity and a comparably high amorphous background, but reveal distinctly different peak patterns and intensities proving them to be different crystal forms to the solvates. However, it can be observed that the desolvated crystal forms of F-TPI and Cl-TPI are isomorphous. This is surprising, as the methanol solvates were not structurally related. $\mathrm{The} \mathrm{Br}-$ TPI and I-TPI desolvated crystal forms, on the other hand, reveal no similarity in their desolvated modification unlike their methanol solvates. It can thus be assumed that the desolvation and concomitant reorganisation process is significantly influenced by the different sizes of the substituent and similar packing arrangements are not energetically preferable. 


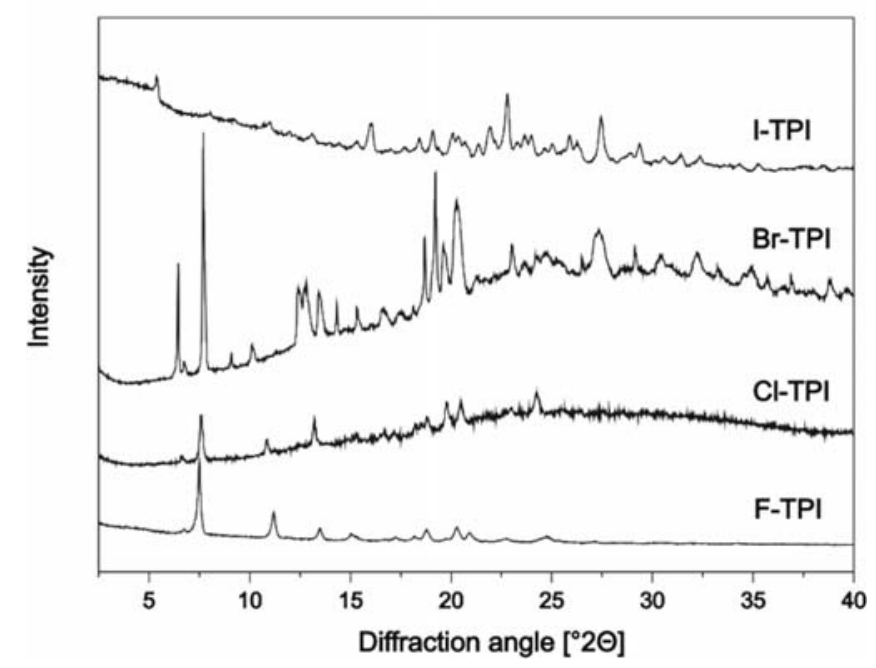

Figure 6. Powder X-ray diffractograms of the crystal forms after desolvation of the methanol monosolvates.

\section{Acetonitrile solvates}

Crystallisation of F-TPI from acetonitrile yielded a crystal form, which TG indicated to be a solvate (Figure $7 \mathrm{a}$ ). The weight loss in the range from 60 to $150{ }^{\circ} \mathrm{C}$ of $3.194 \%$ corresponds well to a stoichiometry of a tetarto-solvate $(0.25 \mathrm{~mol}$ acetonitrile to $1 \mathrm{~mol} \mathrm{F-TPI}$, calculated mass loss: $3.162 \%$ ), whilst the broad desolvation temperature range suggests slow desolvation kinetics. The corresponding differential scanning calorimetry (DSC) trace shows a well-matched desolvation endotherm in the same temperature range followed by a melting endotherm at $252.3{ }^{\circ} \mathrm{C}$ (onset) of the corresponding unsolvated crystal form. 

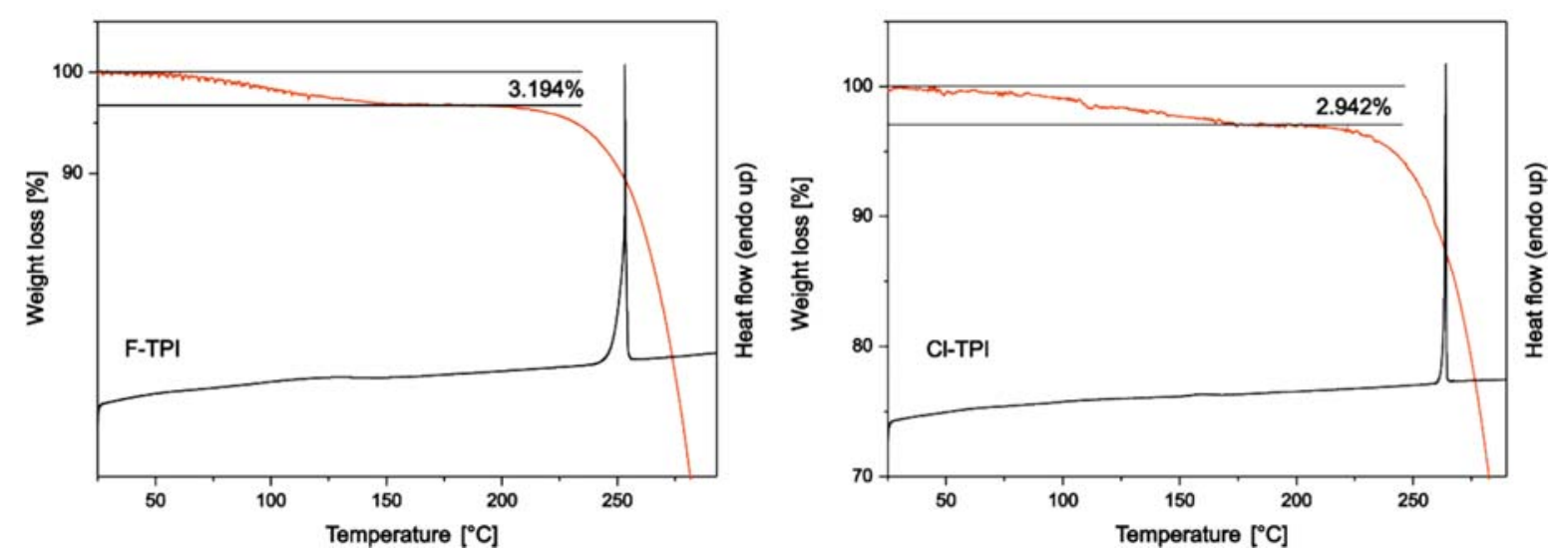

Figure 7. TG and DSC thermograms of acetonitrile solvates of F-TPI and Cl-TPI.

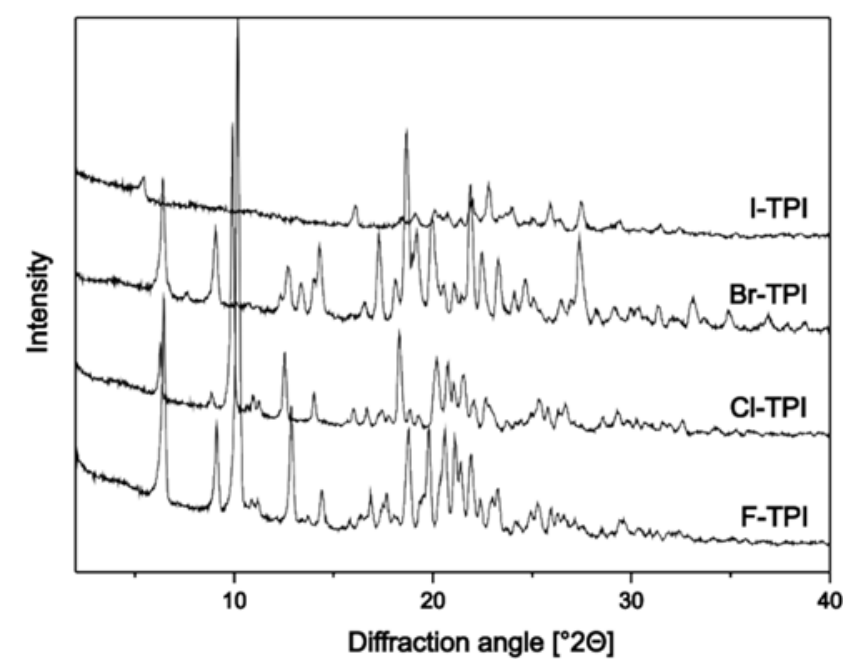

Figure 8. Powder X-ray diffractograms of the crystal forms crystallising from acetonitrile

Changing the TPI substituent from fluorine to chlorine (vdW radius $1.47 \AA$ to $1.77 \AA$ ) seems not to prevent the formation this solvate. Powder X-ray diffraction proved that the Cl-TPI solvate is isostructural to that of F-TPI (Figure 8). The thermal data of the Cl-TPI solvate (Figure 7b) shows a lower mass loss of $2.942 \%$, as detected by TGA, which again corresponds to a tetarto solvate (calculated weight loss $3.009 \%$ ). The desolvation is almost undetectable by DSC, which is probably due to the broad range of this event from $50{ }^{\circ} \mathrm{C}$ to almost $200{ }^{\circ} \mathrm{C}$. The respective heat flow will be so low that it is almost indistinguishable from the baseline. However, a small but 
reproducible endothermic peak can be detected from 150 to $167{ }^{\circ} \mathrm{C}$, which is most likely connected to the desolvation event. Melting subsequently occurs at $262.5^{\circ} \mathrm{C}$.

The change from chlorine to bromine (1.85 $\AA$ van-der-Waals radius) as the substituent proves too big a change for the acetonitrile solvate formation, and the resulting crystal form is unsolvated (Fig. S1). The X-ray powder pattern confirms the presence of a crystal form that is not structurally related to the acetonitrile solvates of F-TPI or Cl-TPI as observable by the distinctly different peak positions. The same is true for iodo-substituted TPI, which shows no detectable weight loss in the TGA measurements (Figure S2) and has distinctly different peak positions in the powder pattern. This I-TPI unsolvated crystal form shows no similarity to any of the other crystal forms from acetonitrile described above for the other halogens

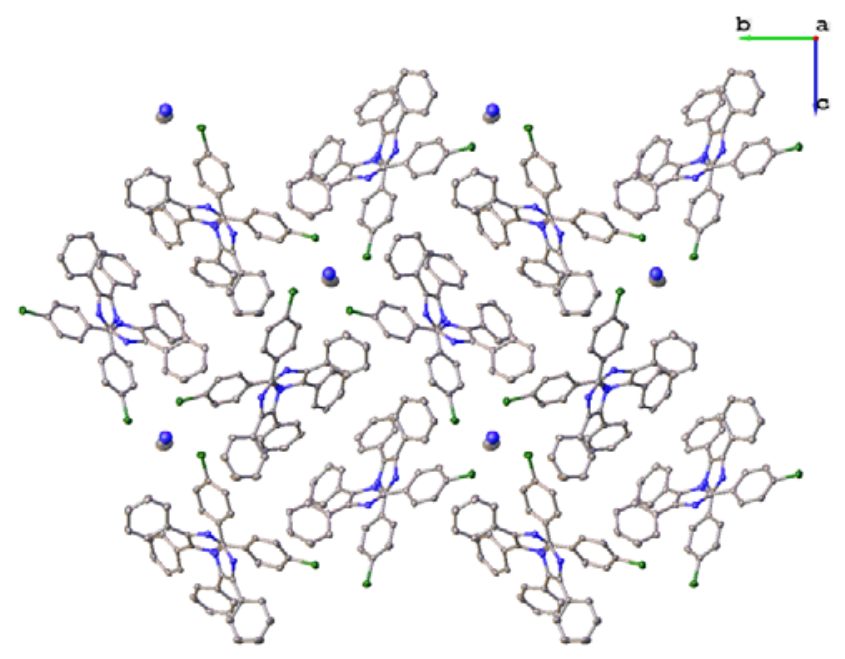

Figure 9. Crystal structure of Cl-TPI acetonitrile solvate along the crystallographic a-axis. Hydrogen atoms omitted for clarity, atomic displacement ellipsoids drawn at 50\%, colour code: carbon (grey), nitrogen (blue), chlorine (green).

Although the investigated solvates of F-TPI and Cl-TPI show a strongly anisotropic crystal growth with a needle-shaped habit, we were able to solve the crystal structure of the Cl-TPI solvate on instrument I19 at the Diamond Light Source, Didcot, UK. The crystal form 
crystallises in the monoclinic spacegroup P $n$ with four molecules of Cl-TPI and one molecule of acetonitrile in the asymmetric unit. The Cl-TPI molecules arrange in the well-reported infinite chains through hydrogen-bonding along the imidazole rings, which are oriented along the crystallographic $a$-axis and also along the preferred axis of growth (Fig. S3). In between four chains of host molecules are channels filled with the solvent, which again is oriented along the crystallographic $a$-axis (Figure 9). These channels are lined alternatingly with a ring of chlorine atoms and a layer of phenyl rings interacting with the nitrogen atom of the solvent through C$\mathrm{H}^{\cdots} \mathrm{N}$ hydrogen bonds, which are generally regarded to be weak. The channels, however, are stabilised by $\mathrm{Cl} \cdots \mathrm{Cl}$ halogen bonds (Fig S4), 3.38-3.63 $\AA$ in length and at a bond angle of 155$162^{\circ}$

The diameter of these pores is approximately $4.93 \AA$, which is only $1.39 \AA$ more than double the van-der-Waals-radius of chlorine. This might explain the desolvation behaviour observed by TGA over a wide temperature range. The channels containing the acetonitrile have a comparable structure to a string of pearls, in which the pearls represent sufficient void space for acetonitrile to diffuse through (i.e. the spaces lined with phenyl rings), whilst the string spaces represent the constriction by the chlorine atoms. When looked at statically, the solvent molecule would not be able to diffuse through these channels and desolvation would be accompanied by destruction of the crystal structure and correlated pseudomorphosis (as seen for the methanol solvates) or at least cracking of the crystal in order to release the solvent. However, as has recently been shown for a porous organic cage structure, crystalline materials are not static and molecules within the structure can move around their position. ${ }^{46}$ The authors of this study show that certain gasses can induce a higher nominal porosity of the material by accessing void space within the structure that is only accessible for very short periods of time. 
Since the F-TPI acetonitrile solvate shows an isostructural powder pattern to Cl-TPI, the crystal structure has the same string-of-pearls channels with constriction through the fluorine atoms. If we assume that the overall crystal structure does not change and the pore size stays approximately the same, the pore diameter would be approximately $2 \AA$ more than the van-derWaals radius of the lining fluorine atoms, and thus present a smaller obstacle to the diffusion of acetonitrile through the pores. This explains the narrower temperature range of the desolvation observed by TGA, whilst the initial stability and higher onset temperature of the weight loss can be explained by the energy necessary to overcome the intermolecular interactions between the solvent and the host molecules in both cases.

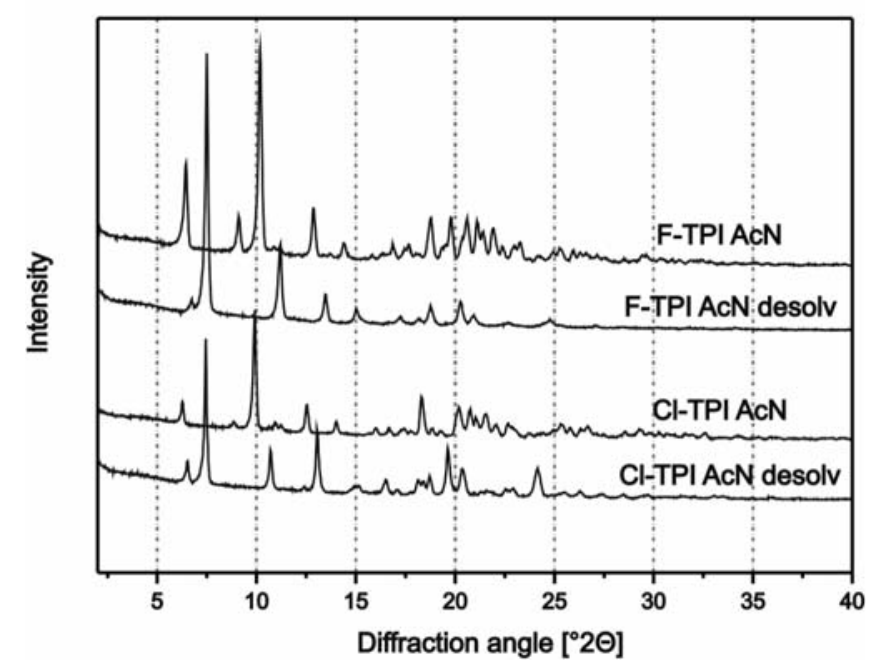

Figure 10. Powder X-ray patterns of acetonitrile solvates and respective desolvates of F-TPI and Cl-TPI. 


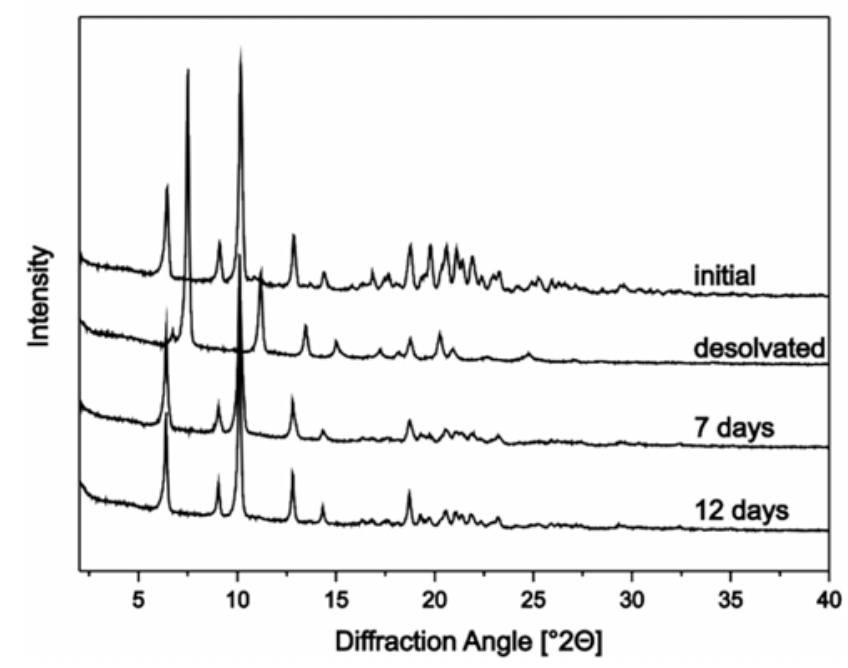

Figure 11. Powder X-ray patterns of solvated, desolvated and resolvated acetonitrile solvate of F-TPI.

In order to show the close relationship of the solvate to the respective desolvated crystal form of F-TPI and Cl-TPI, both solvates were desolvated by heating up to $190{ }^{\circ} \mathrm{C}$ using the TGA and following the weight change to ensure complete desolvation. PXRD analysis confirmed the presence of a new crystal form (Figure 11), which shows peak shift to higher angles and thus smaller $d$-spacings when compared to the original solvated crystal structure. Even though these shifts are comparably large, the overall powder patterns change little. As the solvated crystal forms, the desolvated forms of F-TPI and Cl-TPI are isostructural. Another indication of the isostructurality of both solvates with their respective desolvates is the absence of pseudomorphosis or a moving front indicating single-crystal to single-crystal transition during desolvation, as has been proven by thermo-microscopy. Final proof, however, would only be possible by indexing the powder pattern and solving the crystal structure, which is scope of ongoing work. If the two solvate/desolvate pairs are indeed isostructural, this would corroborate 
our hypothesis of the solvent leaving the crystal structure by diffusion along the channels. Neither of the new crystal forms is related to those observed for Br-TPI or I-TPI.

The unsolvated crystal forms were then stored in a saturated atmosphere of acetonitrile to probe the reversibility of the desolvation process (Figures 11 and S5). After seven days of storage, the PXRD pattern of the materials showed full resolvation to the acetonitrile solvates, whilst storage for a further five days only improves the crystallinity of the sample, as observed by the increased sharpness of the peaks. The resolvation upon exposure to saturated solvent atmosphere could be explained by at least partial retention of the porous structure in the desolvated crystal structure, which contracts in order to fill the voids and thus minimises the crystal lattice energy. This would facilitate the re-uptake of solvent without the necessity of a phase change. Such behaviour has been shown for pharmaceutical hydrates, ${ }^{47,}{ }^{48}$ and can pose problems during manufacturing. In this case, the formation of an isostructural desolvate poses a way to generate a new anhydrous crystal form. ${ }^{17,49}$

Desolvation did not occur when the solvates were stored for 7 days under dry conditions (phosphorous pentoxide) at ambient temperature, which shows that a certain amount of activation energy is necessary to start the desolvation. However, storing the solvate at ambient conditions, i.e. at approximately $50 \%$ relative humidity at $23{ }^{\circ} \mathrm{C}$, led to a transition to the desolvate over a period of two weeks. It is thus likely that water plays an important role in the desolvation of these solvates, and this is the focus of on-going studies.

\section{Unsolvated crystal forms}

Each of the TPI derivatives shows two unsolvated crystal forms, either from desolvation of the solvates or, in the case of Br-TPI and I-TPI, by direct crystallisation from acetonitrile (Fig S6). The F-TPI and Cl-TPI desolvates from both solvates are the same modification. This is 
surprising, as it has been shown above that the acetonitrile solvate and its desolvate are related or even isostructural. It can be assumed that in this case, the desolvate is so stable that it forms during the pseudomorphosis and connected reorganisation of the crystal lattice upon release of methanol from the methanol solvate. In the case of Br-TPI, the desolvate of the methanol solvate results in a different crystal form than direct crystallisation from acetonitrile. This shows the significance of using solvate formation during crystal form discovery, as has been frequently shown for organic compounds. ${ }^{17,20,47,49}$ I-TPI results in the same crystal form independent from the solvent/solvate, which again points towards one energetically favoured modification.

Even though this study shows clearly the influence the simple exchange of one substituent has on the crystallization characteristics of the resulting compounds, the data presented here are only the early stage of the investigation and more information is needed to draw definite conclusions of the substituent influence on the solid-state behaviour. What is blatantly clear is that the simple exchange of a halogen substituent on a simple model compound with reduced complexity can significantly influence the solid-state landscape and the crystal forms available. Whilst the change from fluorine to chlorine seems to have less of an effect, exchanging chlorine with bromine effects a more radical change in the solid-state behaviour. The iodo-substituent then follows the behavior of bromine.

\section{Conclusion}

In conclusion, we have reported the solvate formation of a series of bio-isosterically substituted triphenylimidazoles (2-(4'-p-X-phenyl)-4,5-diphenylimazoles, $\mathrm{X}$-TPI; $\mathrm{X}=\mathrm{F}, \mathrm{Cl}, \mathrm{Br}$, and I) in two industrially used solvents, methanol and acetonitrile. There is a clear difference of solvate formation depending on the size of the substituent as well as the incorporation motif of the solvent. Methanol intercalates into the characteristic hydrogen-bonded imidazole chains, a motif 
that most likely persists independently from the substituent. However, whilst the packing in two dimensions stays the same, the substituent's size has an influence on the packing in the third dimension. Solvate formation from acetonitrile can only be observed for the smaller halogen substituents (F-TPI and Cl-TPI), which form a channel solvate that can be desolvated and resolvated, apparently without changing the overall crystal structure. Since the channels are lined with the halogen substituent, bromine and iodine substitution proves to be too much of a steric hindrance for the packing to be stable, and thus Br-TPI and I-TPI crystallise in non-solvated crystal forms. Two different unsolvated crystal forms can be obtained for Br-TPI by desolvation of the solvates or direct crystallisation from acetonitrile, whilst F-TPI, Cl-TPI and I-TPI crystallise in the same unsolvated crystal form. Overall, there seems to be related behavior between fluoro- and chloro-substitution as well as bromo-and iodo-substitution with little correlation between chloro- and bromo-substitution. This is an interesting finding and should be verified with additional data.

Investigation of this series of model compound for bio-isosteric replacement with reduced complexity shows the significant influence of the size of the substituent on the crystallisation of different modifications. Whilst this can be a big advantage to engineer the appropriate crystal form or particle size, e.g. through desolvation and correlated pseudomorphosis, it can also pose the problem of residual solvent or unwanted solvate formation and thus increase the number of processing steps needed. It is clear from this study that the simple exchange of one substituent for its bio-isostere can have massive influence on the solid-state properties of a compound and that more research into this field is needed.

\section{Associated Content}


Supporting information including additional thermal and crystallographic data available free of charge via the Internet at http://pubs.acs.org. CCDC 1449859, 1477419 and 1477420 contain crystallographic data for this paper. These data can be obtained free of charge via www.ccdc.cam.ac.uk/data_request/cif, or by emailing data_request@ccdc.cam.ac.uk, or by contacting The Cambridge Crystallographic Data Centre, 12, Union Road, Cambridge CB2 1EZ, UK; fax: +44 1223336033 .

\section{Corresponding Author}

* Email: katharina.fucke@durham.ac.uk

\section{Notes}

The authors declare no competing financial interest.

\section{Author Contributions}

The manuscript was written through contributions of all authors. All authors have given approval to the final version of the manuscript.

\section{Acknowledgements}

The authors would like to thank Diamond Light Source, Didcot, UK for the award of instrument time on Station I19 (MT 8682) and the instrument scientist for their kind support.

\section{References}

(1) Showell, G. A.; Mills, J. S., Drug Discovery Today, 2003, 8, 551-556.

(2) Olesen, P. H., Curr. Opin. Drug Discovery Dev., 2001, 4, 471-8.

(3) Mugnaini, C.; Pasquini, S.; Corelli, F., Curr. Med. Chem., 2012, 19, 4794-4815.

(4) Tuyishime, M.; Danish, M.; Princiotto, A.; Mankowski, M. K.; Lawrence, R.; Lombart, H. G.; Esikov, K.; Berniac, J.; Kuang, L.; Ji, J. J.; Ptak, R. G.; Madani, N.; Cocklin, S., Bioorg. Med. Chem. Lett., 2014, 24, 5439-5445. 
(5) Lusher, S. J.; McGuire, R.; van Schaik, R. C.; Nicholson, C. D.; de Vlieg, J., Drug Discovery Today, 2014, 19, 859-868.

(6) Ertl, P., Curr. Opin. Drug Discovery Dev., 2007, 10, 281-288.

(7) Devereux, M.; Popelier, P. L. A., Current Topics in Medicinal Chemistry, 2010, 10, 657668.

(8) Friedman, H. L., NAS-NRS Publication, 1951, 295-358.

(9) Haleblian, J.; McCrone, W., J. Pharm. Sci., 1969, 58, 911-929.

(10) Grant, D. J. W., Theory and origin of polymorphism. In Polymorphism in

Pharmaceutical Solids, Brittain, H. G., Ed. Marcel Dekker Inc.: New York, 1999; Vol. 95, pp 133.

(11) Bernstein, J., Polymorphism in Molecular Crystals. ed.; Clarendon Press: Oxford, 2002.

(12) Brittain, H. G., Polymorphism in Pharmaceutical Solids. ed.; Marcel Dekker Inc.: New York, 1999.

(13) Griesser, U. J., The importance of solvates. In Polymorphism, Hilfiker, R., Ed. WileyVCH: Weinheim, Germany, 2006; pp 211-233.

(14) Fucke, K.; Howard, J. A. K.; Steed, J. W., Chemical Communications, 2012, 48, $12065-$ 12067.

(15) Davey, R. J.; Brychczynska, M.; Sadiq, G.; Dent, G.; Pritchard, R. G., CrystEngComm, 2013, 15, 856-859.

(16) Food and Drug Administration: Guideline Q3C Impurities: Residual Solvent. In ed.; 1997.

(17) Minkov, V. S.; Beloborodova, A. A.; Drebushchak, V. A.; Boldyreva, E. V., Cryst. Growth Des., 2013, 14, 513-522.

(18) Braun, D. E.; Gelbrich, T.; Kahlenberg, V.; Griesser, U. J., CrystEngComm, 2015, 17, 2504-2516.

(19) Zencirci, N.; Griesser, U. J.; Gelbrich, T.; Kahlenberg, V.; Jetti, R. K. R.; Apperley, D. C.; Harris, R. K., J. Phys. Chem. B, 2014, 118, 3267-3280.

(20) Bhattacharya, S.; Saha, B. K., Cryst. Growth Des., 2013, 13, 606-613.

(21) Williams, P. A.; Hughes, C. E.; Buanz, A. B. M.; Gaisford, S.; Harris, K. D. M., The Journal of Physical Chemistry C, 2013, 117, 12136-12145.

(22) Williams, P. A.; Hughes, C. E.; Martin, J.; Courvoisier, E.; Buanz, A. B. M.; Gaisford, S.; Harris, K. D. M., The Journal of Physical Chemistry C, 2016, 120, 9385-9392.

(23) Hirano, S.; Toyota, S.; Toda, F.; Fujii, K.; Uekuasa, H., Angewandte Chemie International Edition, 2006, 45, 6013-6016.

(24) Nassimbeni, L. R.; Bathori, N. B.; Patel, L. D.; Su, H.; Weber, E., Chemical Communications, 2015, 51, 3627-3629.

(25) Samipillai, M.; Nassimbeni, L. R.; Weber, E., J. Chem. Crystallogr., 2014, 44, 293-300.

(26) Jacobs, A.; le Roex, T.; Nassimbeni, L. R.; Toda, F., Org. Biomol. Chem., 2006, 4, 24522457.

(27) Amombo Noa, F. M.; Bourne, S. A.; Su, H.; Nassimbeni, L. R., Cryst. Growth Des., 2016, 16, 1636-1642.

(28) A. Downing, G.; D. MacNicol, D.; S. Frampton, C., Chemical Communications, 1998, 2085-2086.

(29) Inokuma, Y.; Arai, T.; Fujita, M., Nature Chem., 2010, 2, 780-783.

(30) Yoshioka, S.; Inokuma, Y.; Hoshino, M.; Sato, T.; Fujita, M., Chemical Science, 2015, 6, 3765-3768. 
(31) Knepper, I.; Seichter, W.; Skobridis, K.; Theodorou, V.; Weber, E., CrystEngComm, 2015, 17, 6355-6369.

(32) Saha, B. K.; Nangia, A., Chemical Communications, 2005, 3024-3026.

(33) Edkins, R. M.; Hayden, E.; Steed, J. W.; Fucke, K., Chemical Communications, 2015, 51, 5314-5317.

(34) Cote, B.; Boulet, L.; Brideau, C.; Claveau, D.; Ethier, D.; Frenette, R.; Gagnon, M.; Giroux, A.; Guay, J.; Guiral, S.; Mancini, J.; Martins, E.; Masse, F.; Methot, N.; Riendeau, D.; Rubin, J.; Xu, D.; Yu, H.; Ducharme, Y.; Friesen, R. W., Bioorg. Med. Chem. Lett., 2007, 17, 6816-6820.

(35) Edkins, R. M.; Probert, M. R.; Fucke, K.; Robertson, C. M.; Howard, J. A.; Beeby, A., Phys. Chem. Chem. Phys., 2013, 15, 7848-7853.

(36) Edkins, R. M.; Probert, M. R.; Robertson, C. M.; Howard, J. A. K.; Beeby, A., Rsc Advances, 2014, 4, 5351-5356.

(37) Nakashima, K.; Taguchi, Y.; Kuroda, N.; Akiyama, S.; Duan, G. L., Journal of Chromatography-Biomedical Applications, 1993, 619, 1-8.

(38) Thornber, C. W., Chem. Soc. Rev., 1979, 8, 563-580.

(39) Wolkenberg, S. E.; Wisnoski, D. D.; Leister, W. H.; Wang, Y.; Zhao, Z. J.; Lindsley, C. W., Org. Lett., 2004, 6, 1453-1456.

(40) G. M. Sheldrick, CELL_NOW, University of Göttingen, 2003

(41) Blessing, R., Acta Crystallographica Section A, 1995, 51, 33-38.

(42) Dolomanov, O. V.; Bourhis, L. J.; Gildea, R. J.; Howard, J. A. K.; Puschmann, H., J. Appl. Crystallogr., 2009, 42, 339-341.

(43) Sheldrick, G. M., Acta Crystallogr., Sect. A: Found. Crystallogr., 2008, A64, 112-122.

(44) Martinez-Carrera, S., Acta Crystallographica, 1966, 20, 783-789.

(45) Guinier, A.; Bokij, G. B.; Boll-Dornberger, K.; Cowley, J. M.; Durovic, S.; Jagodzinski, H.; Krishna, P.; de Wolff, P. M.; Zvyagin, B. B.; Cox, D. E.; Goodman, P.; Hahn, T.; Kuchitsu, K.; Abrahams, S. C., Acta Crystallographica Section A, 1984, 40, 399-404.

(46) Manurung, R.; Holden, D.; Miklitz, M.; Chen, L.; Hasell, T.; Chong, S. Y.; Haranczyk, M.; Cooper, A. I.; Jelfs, K. E., The Journal of Physical Chemistry C, 2015, 119, 22577-22586.

(47) Stephenson, G. A.; Groleau, E. G.; Kleemann, R. L.; Xu, W.; Rigsbee, D. R., J. Pharm. Sci., 1998, 87, 536-542.

(48) Sundaramurthi, P.; Suryanarayanan, R., J. Pharm. Sci., 2014, 103, 3095-3106.

(49) Iwata, K.; Kojima, T.; Ikeda, Y., Cryst. Growth Des., 2014, 14, 3335-3342. 


\section{$\underline{\text { For Table of Contents use only }}$}

The influence of bio-isosteric replacement on the formation of templating methanol and acetonitrile solvates in lophines

Thomas Kitchen, Connor Melvin, Mohd Nadzri Mohd Najib, Andrei S. Batsanov and Katharina Edkins*

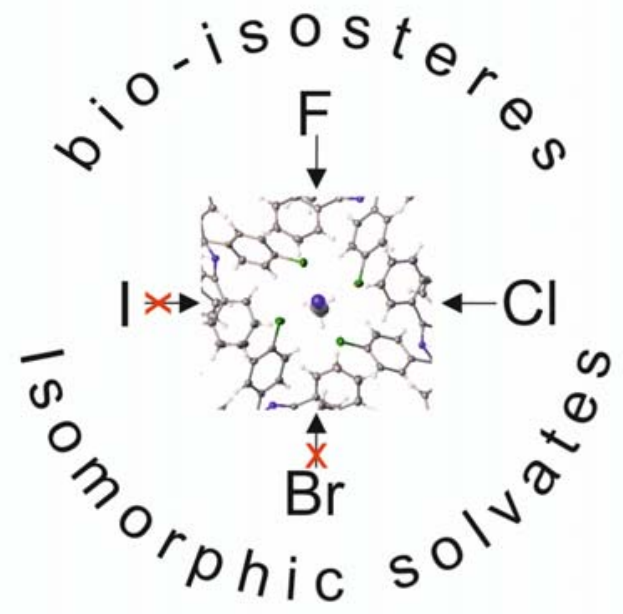

What is the influence of bio-isosteric replacement on the solid-state behaviour of new drug entities? 